\section{Saúde dos adolescentes: um estudo de base populacional em Campinas, São Paulo, Brasil}

\author{
Adolescent health: a population-based study in \\ Campinas, São Paulo State, Brazil
}

\author{
Salud de los adolescentes: un estudio poblacional \\ en Campinas, São Paulo, Brasil
}

Marici Braz ${ }^{1}$

Antonio A. Barros Filho 1

Marilisa B. A. Barros 1

\section{Resumo}

We estimated the prevalence of chronic diseases and other health problems reported by adolescents in relation to social and demographic variables and nutritional status. This cross-sectional population-based survey analyzed data from the Health Survey in Campinas, São Paulo State, Brazil, 2008. We used descriptive statistics and associations between variables with the chisquare test. Prevalence of chronic diseases among adolescents was $19.17 \%$, with asthma showing the highest prevalence (7.59\%), followed by heart disease (1.96\%), hypertension (1.07\%), and diabetes $0.21 \%$. Prevalence rates were $61.53 \%$ for health problems, $40.39 \%$ for allergy, and $24.83 \%$ for frequent headache or migraine. After multivariate analysis using Poisson regression, the factors associated with chronic disease were age 15 to 19 years $(P R=1.38)$, not attending school $(P R=1.46)$, having children $(P R=1.84)$, and obesity $(P R=1.54)$. Female gender $(P R=1.12)$ was statistically associated with health problems. The study illustrates that adolescence is a life stage in which chronic disease and health problems can occur.

Adolescent Health; Chronic Disease;

Epidemiologic Surveys
Estimou-se a prevalência de doenças crônicas diagnosticadas e de problemas de saúde referidos em adolescentes, segundo variáveis sociodemográficas e estado nutricional. É um estudo transversal de base populacional com dados do Inquérito de Saúde do Município de Campinas, São Paulo, Brasil, 2008. Foram utilizadas estatísticas descritivas, teste de associação pelo $\chi^{2}$. A prevalência de doença crônica entre os adolescentes foi de 19,17\%, asma apresentou a maior prevalência 7,59\%, seguida de doenças cardíacas $1,96 \%$, hipertensão $1,07 \%$ e diabetes $0,21 \%$. A prevalência de problemas de saúde foi de 61,53\%, alergia 40,39\% e dor de cabeça frequentelenxaqueca 24,83\% foram mais frequentes. Após análise múltipla por regressão de Poisson os fatores associados à doença crônica foram faixa etária de 15 a 19 anos $(R P=1,38)$, não frequentar escola $(R P=1,46)$, ter filhos $(R P=1,84)$ e ser obeso $(R P=1,54)$, e somente sexo feminino $(R P=1,12)$ se associou a problemas de saúde. A adolescência é uma fase da vida na qual também se adoece.

Saúde do Adolescente; Doença Crônica; Inquéritos Epidemiológicos 


\section{Introdução}

A concepção de que os adolescentes raramente ficam doentes e o hábito de se justificar qualquer problema de saúde, seja físico ou mental, como "normal" ou característico dessa etapa da vida, têm limitado a atenção à saúde dos adolescentes e consequentemente os avanços de pesquisas nessa área ${ }^{1}$. No entanto, na adolescência, fase que se estende por aproximadamente dez anos, o indivíduo está mais vulnerável a determinadas condições e eventos com fortes implicações na saúde como gravidez precoce, início de uso de drogas lícitas e ilícitas, violência nas suas diferentes formas, doenças sexualmente transmissíveis, AIDS 2, entre outras.

O controle das doenças infectocontagiosas, por meio da vacinação, do uso de antibióticos e da melhoria das condições de saneamento básico proporcionou, nas últimas décadas, mudanças significativas no perfil das morbidades. O contexto ambiental e socioeconômico, os hábitos e estilo de vida são fatores determinantes para as condições de saúde das populações. Atualmente, as mudanças dos hábitos alimentares e de atividade física entre crianças e adolescentes têm gerado precocemente agravos à saúde, com aumento de doenças crônicas 3,4 e transtornos mentais 5 .

O aperfeiçoamento do cuidado aos neonatos prematuros, às doenças respiratórias na infância e o aprimoramento das correções cirúrgicas de cardiopatias congênitas contribuíram para o aumento da sobrevida de crianças com maior propensão a doenças crônicas 6 .

As doenças crônicas não transmissíveis normalmente são relacionadas aos adultos e, sobretudo, aos idosos, uma vez que a sua prevalência nesse estrato da população é alta. Em 2003, pela Pesquisa Nacional por Amostra de Domicílios (PNAD/2003), verificou-se que 29,9\% da população brasileira referiam ser portadores de pelo menos uma doença crônica; entre os idosos tal valor atingia $75,5 \% 7$.

A definição de doenças crônicas não transmissíveis proposta pela Organização Mundial da Saúde (OMS) também remete ao adulto, pois seriam doenças que "levam décadas para estar completamente instaladas na vida de uma pessoa e têm origem em idades jovens; sua emergência é em muito influenciada pelas condições de vida e não é resultado unicamente de escolhas individuais" 8 (p. 182), portanto podem acompanhar o indivíduo por longos períodos da vida.

O National Center for Health Statistics, nos Estados Unidos, define de forma mais específica as doenças crônicas na criança e no adolescente como condição com duração superior a três me- ses, que limitam as funções normais da criança e do adolescente e que podem causar hospitalização por mais de um mês 9 . Pode-se acrescentar a tal definição que são agravos permanentes, causados por alterações patológicas irreversíveis e podem exigir longos períodos de supervisão, observação ou cuidados 10 .

Como doença crônica não transmissível, a OMS 8 classifica especialmente as doenças cardiovasculares (cerebrovasculares, isquêmicas), as neoplasias, as doenças respiratórias crônicas e diabetes mellitus. Outras doenças que possam contribuir para o sofrimento dos indivíduos, das famílias e da sociedade, tais como as desordens mentais e neurológicas, as doenças bucais, ósseas e articulares, as desordens genéticas e as patologias oculares e auditivas, também são incluídas nessa classificação.

A carência de dados sobre a saúde dos adolescentes dificulta a determinação da prevalência de doenças crônicas na faixa etária. A justificativa para semelhante dificuldade está ligada à abrangência dos fatores envolvidos na definição de doença crônica em adolescentes. Estão envolvidos: duração, idade de início, se é congênita ou adquirida, limitação de atividades própria da idade, visibilidade, mobilidade, sobrevida esperada, funcionamento fisiológico e sensorial, comprometimento cognitivo, na comunicação ou emocional/social 11, dificultando assim a padronização das pesquisas.

Caraffa \& Sucupira ${ }^{9}$ chamam a atenção para a diferença de conceitos na língua inglesa entre as palavras disease e illness. Disease traz a dimensão objetiva de doença propriamente dita enquanto illness tem a dimensão de sofrimento da subjetividade, que pode ser traduzida pela dor sentida, pela irritação, por sentir-se triste, pelo mal estar ou insônia. Alguns estudos 12 consideram esses problemas de saúde como condições psicossomáticas que também podem ser classificados como doenças crônicas.

Estima-se que a prevalência de doenças crônicas entre crianças e adolescentes nos Estados Unidos seja de $12 \%$ a $16 \%$, percentual que vem crescendo nas últimas décadas 9 . Em alguns países da Europa e no Canadá, avalia-se que cerca de $10 \%$ dos adolescentes sofram com essa condição ${ }^{11}$. No Brasil, a PNAD/2008 revelou que a prevalência de pelo menos uma doença crônica era de $11,2 \%$ nas adolescentes e de $9,5 \%$ nos adolescentes 13 .

Dentre as doenças crônicas, a com maior prevalência entre os adolescentes é a asma e a de maior incidência é o diabetes mellitus, particularmente do tipo II 6 .

Os fatores de risco para doença crônica podem ser divididos em modificáveis e não modi- 
ficáveis. Entre os não modificáveis estão hereditariedade, idade e raça. Entre os modificáveis ou preveníveis estão ingestão abusiva de álcool, tabagismo, sedentarismo, estresse, obesidade e colesterol elevado 14 .

Sabe-se que a adolescência é uma fase da vida de grande exposição a vários dos fatores citados acima, e muitos dos hábitos adquiridos nessa fase da vida são levados para a vida adulta junto com as suas consequências para a saúde 15 .

A relação entre enfermidades crônicas e qualidade de vida vem chamando a atenção nos últimos anos. Em 1998, a OMS definiu qualidade de vida como "a percepção do indivíduo de sua posição na vida, no contexto da cultura e sistema de valores nos quais vive e em relação aos seus objetivos, expectativas, padrões e preocupações" 10 (p. 46). As doenças crônicas podem trazer limitações que irão comprometer a qualidade de vida, sobretudo do adolescente que está em intenso processo de modificações biopsicossociais 10,16.

O desenvolvimento de estratégias que estimulem o aumento da discussão e do planejamento intersetoriais é a proposta da OMS presente no Plano de Ação 2008-2013 para conter as doenças crônicas não transmissíveis 17,18 . No Brasil, o Plano de Ações Estratégicas para o Enfrentamento das Doenças Crônicas Não Transmissíveis 2011-2022 19 prioriza as ações de vigilância, informações e monitoramento das doenças crônicas, assim como as ações de promoção e cuidado com a saúde. Conhecer as condições de saúde dos adolescentes é fundamental para o desenvolvimento de políticas de promoção da saúde e de prevenção de doenças crônicas, que sejam efetivas e sustentáveis. Nessa perspectiva, o objetivo deste trabalho é estimar a prevalência de doenças crônicas e de problemas de saúde nos adolescentes e sua associação com estado nutricional, fatores demográficos e socioeconômicos.

\section{Métodos}

Os dados deste estudo transversal de base populacional foram obtidos de pesquisa de saúde realizado no Município de Campinas, São Paulo, Brasil, em 2008, o Inquérito de Saúde do Município de Campinas (ISA-Camp 2008).

Para esse inquérito, foi tomada uma amostra probabilística por conglomerados, em dois estágios. No primeiro estágio foram sorteados cinquenta setores censitários da área urbana do município. Nesses cinquenta setores, foi feita a listagem dos domicílios particulares existentes. No segundo estágio, o sorteio dos domicílios foi feito de forma a obter mil entrevistas com adolescentes, não institucionalizados, com idade entre
10 e 19 anos. Maiores detalhes do plano amostral podem ser obtidos em Alves 20.

O questionário da pesquisa foi organizado em blocos temáticos com a maioria das questões pré-codificadas e foi aplicado por entrevistadores treinados. As entrevistas foram realizadas diretamente com o adolescente sorteado, exceto em situação de incapacidade do adolescente.

Para garantir a qualidade dos dados coletados, reentrevistas parciais, pessoais ou telefônicas, foram realizadas em amostra aleatória de 5\% dos participantes e não mostraram discrepâncias em relação às informações originais.

Neste estudo, foram utilizadas as informações de morbidade, peso e altura referidos, e informações do domicílio e das características socioeconômicas da família do entrevistado.

Como variáveis dependentes foram estudadas doenças crônicas e problemas de saúde referidos, presentes num checklist, contendo doenças e sintomas de maior relevância na população em geral. Foi questionado ao adolescente se algum médico ou outro profissional de saúde já havia dito alguma vez que ele tinha alguma das seguintes doenças: asma/bronquite/enfisema, doença do coração, problemas de circulação, diabete, hipertensão, tendinite/LER/DORT e reumatismo/ artrite/artrose. Em seguida era perguntado se o adolescente tinha alguns dos seguintes problemas de saúde: alergia, dor de cabeça frequente/ enxaqueca, dor nas costas/problemas na coluna, problema emocional-ansiedade e tristeza, insônia, tontura/vertigem e problemas urinários.

As variáveis independentes do estudo foram: sexo, faixa etária, categorizada de 10 a 14 anos e de 15 a 19 anos, cor da pele, frequência à escola, tipo de escola que frequenta (rede pública ou rede privada de ensino), se trabalha ou só estuda, situação conjugal, gravidez, filhos, renda mensal per capita (em salário mínimo) e escolaridade do chefe de família (em anos de estudo). Também foi analisado o índice de massa corporal (IMC) calculado com dados de peso e altura referidos e para conversão do IMC em estado nutricional, empregaram-se as tabelas do Centers for Disease Control and Prevention (CDC) 21, segundo sexo e idade, classificando-se os adolescentes como baixo peso $(\leq \mathrm{P} 5)$, peso normal $(>\mathrm{P} 5 \mathrm{e} \leq \mathrm{P} 85)$, sobrepeso ( $>$ P85 e $<$ P95) e obesidade ( $\geq$ P95). Apesar de a obesidade ser considerada uma doença crônica, foi avaliada neste estudo como uma variável independente uma vez que não constava do checklist e foi obtida de uma forma indireta pelo cálculo do IMC; logo não foi referida pelo adolescente como doença crônica. Havia também o interesse de analisar a possível associação de doenças crônicas e problemas de saúde com a obesidade. 
Para a análise descritiva, as variáveis contínuas foram transformadas em categóricas e foram calculados as porcentagens e os intervalos de 95\% de confiança tanto para as variáveis dependentes quanto para as independentes.

As associações entre as variáveis dependentes, doenças crônicas e problemas de saúde referidos e as variáveis independentes foram verificadas pelo teste qui-quadrado com nível de significância de 5\%. As razões de prevalência, que são medidas consideradas mais conservadoras e mais facilmente interpretáveis que as razões de odds 22 , foram calculadas por meio de regressão de Poisson. A regressão múltipla de Poisson foi utilizada para proceder ao ajuste por sexo e idade.

As análises estatísticas foram realizadas com o aplicativo Stata 11.0 (Stata Corp., College Station, Estados Unidos) com os comandos svy que permitem incorporar as características do delineamento amostral e as ponderações necessárias.

O comitê de Ética em pesquisa da Faculdade de Ciências Médicas, Universidade Estadual de Campinas (FCM-UNICAMP) aprovou o projeto com o parecer no 079/2007. O termo de consentimento foi lido pelo entrevistador aos entrevistados que confirmaram sua aceitação em participar do estudo. No caso dos adolescentes de 10 a 18 anos, foi solicitada a autorização do responsável.

\section{Resultados}

Entre os domicílios sorteados para obter a amostra de adolescentes, houve $14,8 \%$ de perda provocada por morador ausente ou por recusa do morador em listar os residentes do domicílio. Entre os 955 adolescentes identificados nos domicílios sorteados, 26 recusaram-se a participar do estudo (perda de $2,7 \%$ ) de forma que foram analisados nesta pesquisa os dados de 929 adolescentes. A população estudada tinha o mesmo percentual entre os sexos feminino e masculino, percentual mais elevado de adolescentes na faixa etária de 10 a 14 anos $(54,98 \%)$ e de cor branca $(65,28 \%)$. A maioria frequentava escola (84,6\%), $19,75 \%$ trabalhavam, $2,7 \%$ tinham cônjuge, $3,25 \%$ tinham filhos e $3,42 \%$ das adolescentes estavam grávidas no momento da entrevista. Obesidade foi verificada em $10,17 \%$ dos adolescentes (Tabela 1).

Dos adolescentes do município, 19,17\% referiram ter ao menos uma doença crônica diagnosticada por um profissional de saúde. Dezessete por cento apresentaram apenas uma doença crônica e $1,63 \%$ duas ou mais doenças associadas. Em relação aos problemas de saúde presentes no checklist, $61,53 \%$ dos adolescentes referiram ter algum problema, 33,75\% afirmaram ter um único problema, $17,31 \%$ dois problemas, $7,23 \%$ e 3,25\% referiram ter, respectivamente, três problemas e quatro ou mais problemas de saúde.

Dentre as doenças crônicas, a asma foi a de maior prevalência $(7,59 \%)$, seguida das doenças cardíacas $(1,96 \%)$, sendo 12 adolescentes com sopro cardíaco, três com arritmia cardíaca e dois com prolapso de válvula mitral. Hipertensão apresentou prevalência de $1,07 \%$ e as demais doenças crônico-degenerativas: tendinite, reumatismo, problemas circulatórios e diabetes apresentaram prevalência menor que $1 \%$. Na lista de problemas de saúde referidos os que tiveram maiores prevalências foram: alergia $(40,39 \%)$, dor de cabeça frequente/enxaqueca $(24,83 \%)$, dor nas costas/problema na coluna $(11,84 \%)$ e problemas emocionais como ansiedade e tristeza (10,65\%).

Foram observadas associações estatisticamente significativas entre doença crônica e faixa etária, frequência à escola, situação de trabalho e ter filhos. Após ajuste para faixa etária e sexo, apresentaram prevalências significativamente mais elevadas os adolescentes de 15 a 19 anos, que não frequentavam escola, que tinham filhos e que eram obesos (Tabela 2). Em relação aos problemas de saúde referidos apenas a variável sexo permaneceu associada após o ajuste para a idade (Tabela 3 ).

\section{Discussão}

Este estudo mostra a alta prevalência de doenças crônicas e, sobretudo, de problemas de saúde referidos entre os adolescentes de Campinas, chamando a atenção para a predominância de doenças crônicas nos adolescentes de maior faixa etária e de problemas de saúde nos adolescentes do sexo feminino. Sua relevância está no fato de ser uma pesquisa de base populacional que abrange diversas doenças e problemas de saúde, possibilitando assim ampliar o conhecimento sobre as condições de saúde dos adolescentes.

A dificuldade de avaliar e comparar as prevalências de doenças crônicas em adolescentes é consequência da diversidade dos métodos usados nas pesquisas e da forma como se define doença crônica nessa faixa etária ${ }^{11}$. Neste estudo, verificou-se que entre os adolescentes de Campinas a prevalência de doenças crônicas é de 19,17\% (IC95\%: 15,67-23,22) e de problemas de saúde referidos é de 61,53\% (IC95\%: 54,61-68,02). Tais prevalências foram superiores às encontradas em outras pesquisas. Estudos realizados na França, Suíça e Canadá apontam que 10\% dos 
Características sociodemográficas, econômicas e índice de massa corporal (IMC) de adolescentes do Município de Campinas, São Paulo, Brasil, 2008/2009.

\begin{tabular}{|c|c|c|c|}
\hline Variáveis e categorias & n & $\%$ & IC95\% \\
\hline \multicolumn{4}{|l|}{ Sexo } \\
\hline Masculino & 467 & 50,75 & $47,45-54,05$ \\
\hline Feminino & 462 & 49,25 & $45,55-52,55$ \\
\hline \multicolumn{4}{|l|}{ Faixa etária (anos) } \\
\hline $10-14$ & 511 & 54,98 & $51,22-58,68$ \\
\hline $15-19$ & 418 & 45,02 & $41,32-48,78$ \\
\hline \multicolumn{4}{|l|}{ Raça/Cor da pele } \\
\hline Branca & 598 & 65,28 & $57,29-72,48$ \\
\hline Preta/Parda & 316 & 33,37 & $26,04-41,60$ \\
\hline Amarela/Indígena & 12 & 1,35 & $0,62-2,93$ \\
\hline \multicolumn{4}{|c|}{ Renda familiar per capita (salários mínimos) } \\
\hline$\leq 0,5$ & 304 & 32,46 & $26,06-39,57$ \\
\hline $0,5-1,0$ & 293 & 31,04 & $25,60-37,05$ \\
\hline$>1,0$ & 332 & 36,51 & $1,93-6,03$ \\
\hline \multicolumn{4}{|c|}{ Escolaridade do chefe de família (anos de estudo) } \\
\hline$\leq 4$ & 239 & 25,79 & $19,24-33,63$ \\
\hline $5-8$ & 272 & 29,11 & $23,88-34,97$ \\
\hline $9-12$ & 195 & 21,16 & $16,46-26,77$ \\
\hline$\geq 13$ & 208 & 23,95 & $16,46-33,48$ \\
\hline \multicolumn{4}{|l|}{ Frequenta escola } \\
\hline Sim & 783 & 84,6 & $80,82-87,76$ \\
\hline Não & 145 & 15,4 & $12,24-19,18$ \\
\hline \multicolumn{4}{|l|}{ Trabalha } \\
\hline Não & 735 & 80,25 & $76,30-83,68$ \\
\hline Sim & 183 & 19,75 & $16,32-23,70$ \\
\hline \multicolumn{4}{|l|}{ Estado conjugal } \\
\hline Sem cônjuge & 902 & 97,3 & $95,56-98,37$ \\
\hline Com cônjuge & 26 & 2,7 & $1,63-4,44$ \\
\hline \multicolumn{4}{|l|}{ Filhos } \\
\hline Não & 898 & 96,75 & $94,91-97,86$ \\
\hline Sim & 31 & 3,25 & $2,06-5,09$ \\
\hline \multicolumn{4}{|l|}{ Gestante } \\
\hline Não & 439 & 96,58 & $94,58-97,86$ \\
\hline Sim & 16 & 3,42 & $2,14-5,41$ \\
\hline \multicolumn{4}{|l|}{ IMC } \\
\hline Eutrófico & 575 & 69,95 & $67,09-72,67$ \\
\hline Baixo peso & 30 & 3,63 & $2,39-5,47$ \\
\hline Sobrepeso & 133 & 16,25 & $13,77-19,07$ \\
\hline Obeso & 84 & 10,17 & $8,22-12,50$ \\
\hline
\end{tabular}

IC95\%: intervalo de 95\% de confiança.

adolescentes têm doença crônica e, se asma e deficiência visual forem incluídas, essa prevalência aumenta para $15 \%$. Inquérito escolar realizado com adolescentes europeus verificou que $45,1 \%$ dos estudantes apresentavam algum problema de saúde referido 23 .
A doença de maior prevalência entre as doenças crônicas deste estudo foi a asma $(7,59 \%)$. Índices condizentes foram encontrados em inquéritos com estudantes portugueses, de 11 a 15 anos de idade que apresentavam prevalência de $6,6 \%$ de asma 11,24 e com estudantes de 
Tabela 2

Prevalência de doenças crônicas segundo variáveis sociodemográficas, econômicas e índice de massa corporal (IMC) em adolescentes do Município de Campinas, São Paulo, Brasil, 2008/2009.

\begin{tabular}{|c|c|c|c|}
\hline Variáveis e categorias & Prevalência (IC95\%) & RP bruta (IC95\%) & RP ajustada * (IC95\%) \\
\hline Sexo & $p=0,730 * \star$ & & \\
\hline Masculino & $19,63(15,28-24,85)$ & 1,00 & 1,00 \\
\hline Feminino & $18,69(14,59-23,62)$ & $0,95(0,71-1,26)$ & $0,94(0,71-1,25)$ \\
\hline Faixa etária (anos) & $p=0,012 * \star$ & $p=0,013^{\star \star \star}$ & $p=0,013$ *** \\
\hline $10-14$ & $16,35(12,78-20,69)$ & 1,00 & 1,00 \\
\hline $15-19$ & $22,59(17,97-28,00)$ & $1,38(1,07-1,77)$ & $1,38(1,07-1,77)$ \\
\hline Raça/Cor da pele & $p=0,382 * *$ & & \\
\hline Branca & $18,08(14,40-22,45)$ & 1,00 & 1,00 \\
\hline Preta/Parda & $20,58(15,42-26,92)$ & $1,13(0,84-, 52)$ & $1,15(0,85-1,54)$ \\
\hline Renda familiar per capita (salários mínimos) & $p=0,407 * \star$ & & \\
\hline$\leq 0,5$ & $20,16(14,92-26,67)$ & 1,00 & 1,00 \\
\hline $0,5-1,0$ & $21,02(15,35-28,10)$ & $1,04(0,72-1,49)$ & $1,03(0,73-1,47)$ \\
\hline$>1,0$ & $16,70(12,77-21,54)$ & $0,82(0,57-1,18)$ & $0,80(0,55-1,14)$ \\
\hline Escolaridade do chefe de família (anos de estudo) & $p=0,180$ & & \\
\hline$\leq 4$ & $23,96(17,62-31,70)$ & 1,00 & 1,00 \\
\hline $5-8$ & $17,08(12,38-23,10)$ & $0,71(0,50-1,01)$ & $0,73(0,52-1,02)$ \\
\hline $9-12$ & $16,74(11,62-23,52)$ & $0,69(0,45-1,01)$ & $0,71(0,46-1,10)$ \\
\hline$\geq 13$ & $18,26(13,66-23,99)$ & $0,76(0,50-1,14)$ & $0,76(0,50-1,15)$ \\
\hline Frequenta escola & $p=0,002 * \star$ & 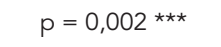 & $p=0,046 \star \star \star *$ \\
\hline Sim & $17,50(14,10-21,53)$ & 1,00 & 1,00 \\
\hline Não & $28,37(21,17-36,87)$ & $1,62(1,21-2,16)$ & $1,46(1,01-2,13)$ \\
\hline Trabalha & $p=0,004 \star \star$ & 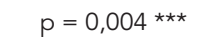 & \\
\hline Não & $17,30(13,93-21,28)$ & 1,00 & 1,00 \\
\hline Sim & $25,09(19,44-31,75)$ & $1,45(1,13-1,85)$ & $1,28(0,90-1,81)$ \\
\hline Estado conjugal & $p=0,280 * *$ & & \\
\hline Sem cônjuge & $18,97(15,47-23,03)$ & 1,00 & 1,00 \\
\hline Com cônjuge & $26,92(13,82-45,04)$ & $1,41(0,76-2,63)$ & $1,23(0,66-2,27)$ \\
\hline Filhos & $p=0,014$ ** & $p=0,009 * \star \star *$ & $\mathrm{p}=0,030$ *** \\
\hline Não & $18,5(14,95-22,67)$ & 1,00 & 1,00 \\
\hline Sim & $38,75(22,87-57,45)$ & $2,09(1,21-3,61)$ & $1,84(1,06-3,18)$ \\
\hline Gestante & $p=0,998 * \star$ & & \\
\hline Não & $18,76(14,84-23,43)$ & 1,00 & 1,00 \\
\hline Sim & $18,74(6,27-44,28)$ & $0,99(0,39-2,5)$ & $0,90(0,35-2,30)$ \\
\hline IMC & $p=0,140 * *$ & & $p=0,046 * \star \star$ \\
\hline Eutrófico & $19,33(15,13-24,36)$ & 1,00 & 1,00 \\
\hline Baixo peso & $20,51(8,72-41,07)$ & $1,06(0,49-2,28)$ & $1,10(0,50-2,41)$ \\
\hline Sobrepeso & $13,14(8,16-20,48)$ & $0,67(0,41-1,11)$ & $0,73(0,44-1,21)$ \\
\hline Obeso & $26,87(16,40-40,77)$ & $1,39(0,89-2,16)$ & $1,54(1,01-2,37)$ \\
\hline
\end{tabular}

IC95\%: intervalo de 95\%; de confiança; RP: razão de prevalência.

* RP ajustada para faixa etária e sexo;

** Valor de $p$ do teste $\chi^{2}$;

*** Valor de $\mathrm{p}$ da regressão de Poisson.

Ceilândia no Distrito Federal, com idade entre 12 e 18 anos, que apresentavam prevalência de $7,9 \%$ 1. Outros dois estudos apresentaram percentuais mais elevados de asma: no estudo da coorte dos nascidos em 1982 em Pelotas, Rio Grande do Sul, Victora et al. 25, em 2000, observaram que $18,6 \%$ dos adolescentes do sexo masculino com 18 anos referiam ter asma, e no inqué- 
Prevalência de problemas de saúde referidos segundo variáveis sociodemográficas, econômicas e índice de massa corporal (IMC) em adolescentes do Município de Campinas, São Paulo, Brasil, 2008/2009.

\begin{tabular}{|c|c|c|c|}
\hline Variáveis e categorias & Prevalência (IC95\%) & RP bruta (IC95\%) & RP ajustada * (IC95\%) \\
\hline Sexo & $p=0,029 \star \star$ & $p=0,027^{\star \star \star \star}$ & $p=0,027 * \star \star$ \\
\hline Masculino & $57,49(50,34-64,35)$ & 1,00 & 1,00 \\
\hline Feminino & $64,60(56,57-71,89)$ & $1,12(1,01-1,24)$ & $1,12(1,01-1,24)$ \\
\hline Faixa etária (anos) & $p=0,494$ * & & \\
\hline $10-14$ & $59,77(50,90-68,06)$ & 1,00 & 1,00 \\
\hline $15-19$ & $62,48(55,83-68,70)$ & $1,04(0,91-1,19)$ & $1,04(0,91-1,19)$ \\
\hline Raça/Cor da pele & $p=0,427$ ** & & \\
\hline Branca & $62,09(55,81-67,98)$ & 1,00 & 1,00 \\
\hline Preta/Parda & $58,29(47,02-68,76)$ & $0,93(0,79-1,10)$ & $0,94(0,79-1,10)$ \\
\hline Renda familiar per capita (salários mínimos) & $p=0,232$ ** & & \\
\hline$\leq 0,5$ & $55,39(47,84-62,70)$ & 1,00 & 1,00 \\
\hline $0,5-1,0$ & $62,72(50,36-73,62)$ & $1,13(0,93-1,36)$ & $1,12(0,93-1,35)$ \\
\hline$>1,0$ & $64,51(55,50-72,59)$ & $1,16(0,98-1,38)$ & $1,16(0,98-1,37)$ \\
\hline Escolaridade do chefe de família (anos de estudo) & $p=0,337$ & & \\
\hline$\leq 4$ & $66,84(60,08-72,97)$ & 1,00 & 1,00 \\
\hline $5-8$ & $59,88(47,68-70,97)$ & $0,89(0,73-1,09)$ & $0,88(0,72-1,07)$ \\
\hline $9-12$ & $56,64(47,00-65,80)$ & $0,84(0,71-1,01)$ & $0,84(0,71-1,00)$ \\
\hline$\geq 13$ & $64,49(51,92-70,24)$ & $0,91(0,78-1,07)$ & $0,92(0,78-1,07)$ \\
\hline Frequenta escola & $p=0,160 * *$ & & \\
\hline Sim & $60,02(52,64-66,97)$ & 1,00 & 1,00 \\
\hline Não & $66,10(57,46-73,78)$ & $1,10(0,96-1,25)$ & $1,10(0,96-1,25)$ \\
\hline Trabalha & $p=0,303 * *$ & & \\
\hline Não & $59,77(51,81-67,26)$ & 1,00 & 1,00 \\
\hline $\operatorname{Sim}$ & $65,23(56,25-73,24)$ & $1,09(0,92-1,20)$ & $1,09(0,92-1,28)$ \\
\hline Estado conjugal & $p=0,086$ ** & $p=0,026 * * \star$ & $p=0,053 * \star \star$ \\
\hline Sem cônjuge & $60,51(53,54-67,09)$ & 1,00 & 1,00 \\
\hline Com cônjuge & $80,68(56,77-93,00)$ & $1,33(1,03-1,71)$ & $1,28(0,99-1,65)$ \\
\hline Filhos & $p=0,638$ ** & & \\
\hline Não & $60,87(53,82-67,48)$ & 1,00 & 1,00 \\
\hline Sim & $64,80(48,34-78,36)$ & $1,06(0,82-1,37)$ & $1,01(0,78-1,31)$ \\
\hline Gestante & $p=0,878 * *$ & & \\
\hline Não & $64,11(55,93-71,55)$ & 1,00 & 1,00 \\
\hline Sim & $62,50(40,19-80,53)$ & $0,97(0,69-1,36)$ & $0,97(0,69-1,36)$ \\
\hline IMC & $p=0,332$ ** & & \\
\hline Eutrófico & $64,59(57,78-70,85)$ & 1,00 & 1,00 \\
\hline Baixo peso & $67,05(41,11-85,57)$ & $1,03(0,72-1,48)$ & $1,05(0,73-1,53)$ \\
\hline Sobrepeso & $55,27(43,64-66,35)$ & $0,85(0,70-1,03)$ & $0,86(0,71-1,03)$ \\
\hline Obeso & $59,64(49,56-68,96)$ & $0,92(0,76-1,11)$ & $0,94(0,77-1,14)$ \\
\hline
\end{tabular}

IC95\%: intervalo de 95\%; de confiança; RP: razão de prevalência.

* RP ajustada para faixa etária e sexo;

** Valor de $p$ do teste $\chi^{2}$

*** Valor de $\mathrm{p}$ da regressão de Poisson.

rito de saúde de escolares americanos YRBSS de 2009 verificou-se que $22 \%$ (IC95\%: 20,8-23,1) dos estudantes entre os 9o e 12 o anos de escolaridade já haviam apresentado asma alguma vez na vida, e que $10,8 \%$ (IC95\%: 9,9-11,7) apresentavam asma no momento da pesquisa 26. Já o PNAD/2008 apresentou um percentual mais baixo $(5,48 \%)$ de asma entre os adolescentes brasileiros 13 . 
Hipertensão arterial $(1,07 \%)$ e diabetes $(0,21 \%)$ apresentaram baixa prevalência entre os adolescentes de Campinas. Valores próximos a esses foram encontrados no PNAD/2008 13. A baixa prevalência de diabetes foi constatada também entre os estudantes portugueses $(0,5 \%) 24$, no entanto pesquisa com adolescentes do ensino médio, na cidade de Recife, Pernambuco, encontrou a prevalência de $17,3 \%$ de pressão arterial elevada entre estudantes de 14 a 20 anos 27 .

Alergia $(40,39 \%)$ e dores de cabeça frequentes/enxaqueca $(24,83 \%)$ apareceram como os problemas de saúde mais frequentes. Esses percentuais são superiores aos relatados em estudos nacionais que apontam $27,3 \%$ de alergias1 e aos estudos internacionais que apontam $13,5 \%$ de alergia e 9,4\% de dor de cabeça 11,24 .

A prevalência de obesidade neste estudo $(10,17 \%)$ foi maior que a encontrada no ISACamp 2001/2002, que apresentava apenas 3,5\% dos adolescentes com obesidade 28 , dado que demonstra o aumento da obesidade na cidade de Campinas, situação semelhante à que vem ocorrendo em outros países.

Comparando as prevalências de obesidade e sobrepeso nos adolescentes de Campinas com as de estudos nacionais e internacionais, pode-se verificar que a prevalência de obesidade $(10,17 \%)$ foi superior à encontrada na Pesquisa Nacional de Saúde do Escolar (PeNSE) 2 realizada nas capitais brasileiras no ano de 2009, que apresentou média nacional de obesidade igual a $7,2 \%$. Já a média nacional de sobrepeso da PeNSE 2 (16\%) foi semelhante à encontrada neste trabalho. Em Portugal, a prevalência é bastante inferior à do presente estudo e apresentou aumento discreto entre os anos de 2006 e 2010, passando de $2,8 \%$ para $3,4 \%$, e a prevalência de sobrepeso se manteve inalterada e foi próxima a 15\% 11,24. Nos Estados Unidos, em 2009, observaram-se, entre escolares do Ensino Médio, prevalências de obesidade igual a $12 \%$ (IC95\%: 10,9-13,1) e de sobrepeso igual a 15,8\% (IC95\%: 14,7-17,0) 26 . Considerando os intervalos de confiança, podese afirmar que são iguais às prevalências encontradas nos adolescentes de Campinas.

Neste estudo, observou-se que os adolescentes na faixa etária de 15 a 19 anos têm 1,38 vez mais chance de apresentar doença crônica que aqueles da faixa etária de 10 a 14 anos. Tal tendência também foi encontrada nos inquéritos realizados pelo sistema de vigilância de fatores de risco à saúde dirigida a adolescentes: Health Behaviour in School-aged Children (HBSC) em países europeus 11,23.

A presença de ao menos um problema de saúde não apresentou associação com a faixa etária, mas as adolescentes de Campinas apre- sentaram $12 \%$ mais chance de ter problema de saúde do que os adolescentes do sexo masculino, fenômeno que também se reproduz com a população adulta 29 e com adolescentes de outras pesquisas 11,23,30. Esse achado pode ser justificado pelo fato de o sexo feminino estar associado à pior avaliação do seu estado de saúde e pelo fato de os homens, e por conseguinte os rapazes, serem menos propensos a revelarem os seus problemas, portanto teriam menos queixas 30 .

A associação entre doença crônica e trabalho deixa de ser estatisticamente significante após ajuste pera a idade, e confirma-se que a prevalência de adolescentes com doença crônica que não frequentam escola é 1,46 vez maior do que aqueles que vão à escola, e dos que têm filhos é de 1,84 vez maior comparado àqueles que não têm filhos, mesmo após ajuste para a idade.

Choquet et al. 31, num estudo comparativo, encontraram maior prevalência de gravidez e de doenças sexualmente transmissíveis entre os adolescentes franceses com doenças crônicas e deficiências físicas. Em recente revisão bibliográfica, Manfré et al. 32 constataram que as adolescentes grávidas e com filhos são mais sujeitas a sintomas de ansiedade e depressão e ao uso de tabaco.

Como esperado, já que obesidade é fator de risco para diversas doenças como hipertensão, diabetes mellitus entre outras 33 , verificou-se associação estatística significante do IMC acima do percentil 95 com a presença de doença crônica.

Desigualdades sociais, ao contrário do que são observas em estudos com adultos e crianças $34,35,36$, não foram evidenciadas nesta pesquisa. Não foi verificada associação estatística das doenças crônicas e de problemas de saúde com as variáveis: renda familiar, escolaridade do chefe de família e raça/cor da pele, revelando que as elevadas prevalências de doenças crônicas e de problemas de saúde atingem os adolescentes de todos os segmentos sociais. Como em vários outros estudos realizados com adolescentes, observou-se aqui a equalização das desigualdades em saúde na adolescência 37,38 . Não há uma compreensão clara para esse fato, e muitos autores questionam se realmente a adolescência representa um período de equalização relativo de saúde 37 . Estima-se que exista dificuldade em obter dos adolescentes informações corretas sobre as condições socioeconômicas da família; diversos estudos mostram que entre $20 \%$ e $45 \%$ dos adolescentes não sabem informar qual a ocupação dos pais.

Solicitar ajuda aos familiares em caso de dúvidas na hora de responder ao questionário é uma das vantagens da pesquisa domiciliar, assim 
como a possibilidade de o entrevistador avaliar as reais condições socioeconômicas do adolescente, tornando os dados mais consistentes.

A ausência de disparidades socioeconômicas na prevalência de doenças e problemas de saúde de adolescentes difere dos resultados de pesquisas desenvolvidas no mesmo município em outras faixas etárias. Significativas desigualdades na presença de doenças e de fatores de risco foram detectadas em Campinas no segmento de idosos 39 , e em homens 40 e mulheres 41 adultos. Porém, nesses estudos, os autores chamam a atenção para a presença de relativa equidade quanto a vários indicadores de acesso a serviços de saúde, o que sinaliza a boa cobertura oferecida pelos serviços públicos de saúde do município.

A maioria das pesquisas nacionais e internacionais com adolescentes acontece no ambiente escolar 42 , é feita com questionários autoadministrados e é realizada em séries escolares especificas, selecionando determinada faixa etária. Os inquéritos domiciliares possibilitam a inclusão daqueles adolescentes que não estão na escola seja por absenteísmo causado por uma doença crônica, seja por evasão para inserção no mercado de trabalho ou por gravidez precoce, contudo não permite incluir pacientes com doenças crônicas graves que requerem hospitalizações prolongadas, uma vez que os inquéritos domiciliares não contemplam pessoas institucionalizadas. A pesquisa no domicílio possibilita uma amostra com distribuição etária mais ampla, englobando indivíduos de 10 a 19 anos. Neste estudo, verificou-se que $15,4 \%$ dos adolescentes de Campinas não frequentavam mais a escola, e foram incluídos no estudo por se tratar de um inquérito domiciliar.
Dentre as limitações deste estudo, deve-se considerar que a prevalência das doenças crônicas referidas pode ter sido subestimada pela ausência de um diagnóstico. Visando minimizar algum esquecimento, foi utilizado um checklist contendo as doenças crônicas e os problemas de saúde e o preenchimento dos questionários foram realizados por entrevistadores treinados; com isso, garantiu-se que as informações fossem mais precisas do que as obtidas por meio de questionário autoadministrado 34 .

Outra limitação que pode ser apontada é a validade das informações dadas pelos próprios adolescentes sobre peso e altura. Outros estudos já verificaram a concordância desses dados $43 \mathrm{e}$ consideraram que as informações de peso e altura referidas pelos entrevistados, tanto adultos quanto adolescentes, têm boa acurácia para estudos epidemiológicos 44,45.

A elevada prevalência $(61,53 \%)$ de adolescentes com problemas de saúde encontrada neste estudo chama a atenção. Dor de cabeça frequente, dor nas costas, problemas emocionais, insônia e tontura podem ser sintomas resultantes de situações estressantes vivenciadas pelos adolescentes ou influenciadas pelo contexto social no qual o adolescente está inserido. No entanto também podem ser sintomas, muitas vezes pouco valorizados pelos profissionais de saúde, que indiquem uma doença crônica que não foi diagnosticada e dessa forma devem ser mais bem avaliados. Portanto, tal achado alerta para que se reveja a ideia de que a adolescência é uma fase da vida na qual pouco se adoece. 


\section{Resumen}

Se estimó la prevalencia de enfermedades crónicas y problemas de salud informados por los adolescentes. de acuerdo con las variables sociodemográficas y su estado nutricional. Se trata de un estudio transversal de base poblacional con datos de la Encuesta de Salud de Campinas, São Paulo, Brasil, 2008. Se utilizó estadística descriptiva con el test de asociación de $\chi^{2}$. La prevalencia de enfermedad crónica entre adolescentes fue de un 19,17\%, el asma presentó la mayor prevalencia un 7,59\%, seguido por la enfermedad cardíaca un 1,96\%, hipertensión un 1,07\% y la diabetes un 0,21\%. La prevalencia de problemas de salud fue de un 61,53\%, la alergia un 40,39\% y frecuentes dolores de cabeza/migraña un 24,83\% fueron los problemas de salud más frecuentes. Tras el análisis multivariante, mediante la regresión de Poisson, los factores asociados con la enfermedad crónica tenían entre 15 y 19 años $(R P=1,38)$, no asisten a la escuela $(P R=1,46)$, tienen hijos $(R P=1,84)$ $y$ obesidad $(R P=1,54), y$ sólo el sexo femenino $(R P=$ $1,12)$ se asoció con problemas de salud. La adolescencia es una etapa de la vida en la que también se enferma.

Salud del Adolescente; Enfermedad Crónica; Encuestas Epidemiológicas

\section{Colaboradores}

M. Braz participou da revisão da literatura, análise e interpretação dos dados e redação do artigo. A. A Barros Filho orientou a proposta do artigo, participou da análise dos dados e redação do artigo. M. B. A. Barros orientou a proposta do artigo, participou da análise dos dados, redação do artigo e coordenou a pesquisa de campo.

\section{Agradecimentos}

Os autores agradecem ao CNPq (projeto no 409747/2006-8) pelo financiamento da pesquisa e pela bolsa de produtividade científica de M. B. A. Barros. Ao Ministério da Saúde e à Secretaria de Saúde de Campinas pelo apoio financeiro para realização da pesquisa (UNICAMP/Funcamp/SMS, no 4.300). Ao Roberto Celso Colacioppo e à Priscila Maria Stolses Bergamo Francisco pelo apoio nas análises estatísticas.

\section{Referências}

1. Souza EM, Abrão FPS, Motta IA, Almeida JO. Autopercepção do estado de saúde: um estudo de prevalência com adolescentes de Ceilândia, Distrito Federal, Brasil. Comun Ciênc Saúde 2006; 17:9-15.

2. Malta DC, Sardinha LMV, Mendes I, Barreto SM, Giatti L, Castro IRR, et al. Prevalência de fatores de risco e proteção de doenças crônicas não transmissíveis em adolescentes: resultados da Pesquisa Nacional de Saúde do Escolar (PeNSE), Brasil, 2009. Ciênc Saúde Coletiva 2010; 15 Suppl 2: 3009-19.

3. Gabbay M, Cesarini PR, Dib SA. Diabetes melito do tipo 2 na infância e adolescência: revisão da literatura. J Pediatr (Rio J.) 2003; 79:201-8.

4. Camilo DF, Ribeiro JD, Toro ADC, Baracat ECE, Barros Filho AA. Obesidade e asma: associação ou coincidência? J Pediatr (Rio J.) 2010; 86:6-14.
5. Organização Pan-Americana da Saúde. Doenças crônico-degenerativas e obesidade: estratégia mundial sobre alimentação saudável, atividade física e saúde. Brasília: Organização Pan-Americana da Saúde; 2003.

6. Viner R, Booy R. ABC of adolescence: epidemiology of health and illness. BMJ 2005; 330:411-4.

7. Instituto Brasileiro de Geografia e Estatística. Indicadores sociodemográficos e de saúde no Brasil 2009. Rio de Janeiro: Instituto Brasileiro de Geografia e Estatística; 2009. (Série Estudos e Pesquisas. Informação Demográfica e Socioeconômica, 25).

8. World Health Organization. Preventing chronic diseases a vital investment. Geneva: World Health Organization; 2005. 
9. Caraffa RC, Sucupira ACSL. Papel do pediatra geral nas condições crônicas de saúde. In: Sucupira ACS, Kobinger MEBA, Saito MI, Bourroul MLM, Zuccolotto SMC, organizadores. Pediatria em consultório. 5a Ed. São Paulo: Editora Sarvier; 2010. p. 184-9.

10. Masson VA, Monteiro MI, Vedovato TG. Qualidade de vida e instrumentos para avaliação de doenças crônicas: revisão de literatura. In: Vilarta R, Gutierrez GL, Monteiro MI, organizadores. Qualidade de vida: evolução dos conceitos e práticas no século XXI. Campinas: Instituto de Pesquisas Especiais para a Sociedade; 2011. p. 45-54.

11. Suris JC, Michaud PA, Viner R. The adolescent with a chronic condition. Part I: developmental issues. Arch Dis Child 2004; 89:938-42.

12. Aventura Social. Aventura social \& saúde indicadores de saúde dos adolescentes portugueses relatório preliminar HBSC. http://aventurasocial. com/2005/main.php (acessado em 18/Out/2008).

13. Barros MBA, Francisco PMSB, Zanchetta LM, Cesar CLG. Tendências das desigualdades sociais e demográficas na prevalência de doenças crônicas no Brasil, PNAD: 2003-2008. Ciênc Saúde Coletiva 2011; 16:3755-68.

14. Casado L, Vianna LM, Thuler LCS. Fatores de risco para doenças crônicas não transmissíveis no Brasil: uma Revisão sistemática. Rev Bras Cancerol 2009; 55:379-88.

15. Salonna F, van Dijk JP, Geckova AM, Sleskova M, Groothoff JW, Reijneveld AS. Social inequalities in changes in health-related behaviour among Slovak adolescents aged between 15 and 19: a longitudinal study. BMC Public Health 2008; 8:57.

16. Minayo MCS, Hartz ZMA, Buss PM. Qualidade de vida e saúde: um debate necessário. Ciênc Saúde Coletiva 2000; 5:7-18.

17. Secretaria de Atenção à Saúde, Secretaria de Vigilância à Saúde, Ministério da Saúde. Diretrizes e recomendações para o cuidado integral de doenças crônicas não transmissíveis: promoção da saúde, vigilância, prevenção e assistência. Brasília: Ministério da Saúde; 2008. (Série Pactos pela Saúde).

18. Schmidt MI, Duncan BB, Silva GA, Menezes AM, Monteiro CA, Barreto SM, et al. Doenças crônicas não transmissíveis no Brasil: carga e desafios atuais. (Saúde no Brasil, 4). http://download.thelan cet.com/flatcontentassets/pdfs/brazil/brazilpor4. pdf (acessado em 09/Mai/2011).

19. Departamento de Análise de Situação de Saúde, Secretaria de Vigilância em Saúde, Ministério da Saúde. Plano de ações estratégicas para o enfrentamento das doenças crônicas não transmissíveis (DCNT) no Brasil, 2011-2022. Brasília: Ministério da Saúde; 2011.

20. Alves MCGP. Isa-Campinas 2008/09: plano de amostragem. http://www.fcm.unicamp.br/cen tros/ccas/arquivos/plano_de_amostragem.pdf (acessado em 04/Mar/2011).

21. Kuczmarski RJ, Ogden CL, Guo SS, GrummerStrawn LM, Flegal KM, Mei Z, et al. 2000 CDC growth charts for the United States: methods and development. Vital Health Stat 11 2002; (246):147-8.
22. Thompson ML, Myers JE, Kriebel D. Prevalence odds ratio or prevalence ratio in the analysis of cross sectional data: what is to be done? Occup Environ Med 1998; 55:272-7.

23. Currie C, Gabhainn SN, Godeau E, Roberts C, Smith R, Currie D, et al. Inequalities in young people's health HBSC international report from the 2005/2006 survey. Geneva: World Health Organization; 2008.

24. Aventura Social. HBSC: Health Behaviour in Schoolaged Children. Problemas emergentes e contextos sociais dados nacionais 2010-abril 2011. http:// aventurasocial.com/arquivo/1302897373_2b-HB SC\%20Adolescentes\%202010-11.pdf (acessado em 18/Abr/2011).

25. Victora CG, Barros FC, Lima RC, Behague DP, Gonçalves H, Horta BL, et al. The Pelotas Birth Cohort Study, Rio Grande do Sul, Brazil, 1982-2001. Cad Saúde Pública 2003; 19:1241-56.

26. Eaton DK, Kann L, Kinchen S, Shanklin S, Ross J, Hawkins J, et al. Youth risk behavior surveillance United States, 2009. MMWR Surveill Summ 2010; 59:1-142.

27. Gomes BMR, Alves JGB. Prevalência de hipertensão arterial e fatores associados em estudantes de Ensino Médio de escolas públicas da Região Metropolitana do Recife, Pernambuco, Brasil, 2006. Cad Saúde Pública 2009; 25:375-81.

28. Cocetti M, Barros MBA. Prevalência de obesidade na adolescência. In: Barros MBA, César CLG, Carandina L, Goldbaum M, organizadores. As dimensões da saúde: inquérito populacional em Campinas. São Paulo: Editora Hucitec; 2008. p. 135-42.

29. Carandina L. Morbidade referida. In: Barros MBA, César CLG, Carandina L, Goldbaum M, organizadores. As dimensões da saúde: inquérito populacional em Campinas. São Paulo: Editora Hucitec; 2008. p. 113-23.

30. Claro LBL, March C, Mascarenhas MTM, Castro IAB, Rosa MLG. Adolescentes e suas relações com serviços de saúde: estudo transversal em escolares de Niterói, Rio de Janeiro, Brasil. Cad Saúde Pública 2006; 22:1565-74.

31. Choquet M, Fediaevsky LP, Manfredi R. Sexual behavior among adolescents reporting chronic conditions: a French national survey. J Adolesc Health 1997; 20:62-7.

32. Manfré CC, Queiróz SG, Matthes ACS. Considerações atuais sobre gravidez na adolescência. Rev Bras Med Fam Comunidade 2010; 5:48-54.

33. Magalhães VC, Mendonça GAS. Prevalência e fatores associados a sobrepeso e obesidade em adolescentes de 15 a 19 anos das regiões Nordeste e Sudeste do Brasil, 1996 a 1997. Cad Saúde Pública 2003; 19 Suppl 1:S129-39.

34. Barros MBA, César CLG, Carandina L, Torre GD. Desigualdades sociais na prevalência de doenças crônicas no Brasil, PNAD-2003. Ciênc Saúde Coletiva 2006; 11:911-26.

35. Institute of Community Health Sciences. Health of young peoples in East London: The RELACHS Study 2001. London: Institute of Community Health Sciences; 2003. 
36. Barreto SM, Giatti L, Hernaez AM. Family factors associated with negative assessment of children's health. Eur J Public Health 2011; 21:649-55.

37. West P, Sweeting H. Evidence on equalisation in health in youth from the West of Scotland. Soc Sci Med 2004; 59:13-27.

38. Friestad C, Klepp KI. Socioeconomic status and health behavior patterns through adolescence: results from a prospective cohort study in Norway. Eur J Public Health 2006; 16:41-7.

39. Barros MBA, Francisco PMSB, Lima MG, César CLG. Social inequalities in health among the elderly. Cad Saúde Pública 2011; 27 Suppl 2:S198-208.

40. Bastos TF, Alves MCGP, Barros MBA, César CLG. Men's health: a population-based study on social inequalities. Cad Saúde Pública 2012; 28:2133-42.

41. Senicato C, Barros MBA. Social inequality in health among women in Campinas, São Paulo State, Brazil. Cad Saúde Pública 2012; 28:1903-14.
42. Castro IRR, Cardoso LO, Engstrom EM, Levy RB, Monteiro CA. Vigilância de fatores de risco entre adolescentes. Cad Saúde Pública 2008; 24:2279-88.

43. Fonseca MJM, Faerstein E, Chor D, Lopes CS. Validade de peso e estatura informados e índice de massa corporal: estudo Pró-saúde. Rev Saúde Pública 2004; 38:392-8.

44. Peixoto MRG, Benício MHD, Jardim PCBV. Validade do peso e da altura autorreferidos: o estudo de Goiânia. Rev Saúde Pública 2006; 40:1065-72.

45. Farias Júnior JC. Validade das medidas autorreferidas de peso e estatura para o diagnóstico do estado nutricional de adolescentes. Rev Bras Saúde Matern Infant 2007; 7:167-74.

Recebido em 25/Nov/2012

Versão final reapresentada em 26/Fev/2013

Aprovado em 27/Mar/2013 